\title{
The diameter of the symplectomorphism group is infinite
}

\author{
Yakov Eliashberg ${ }^{1, \star}$ and Tudor Ratiu ${ }^{2, \star \star}$ \\ ${ }^{1}$ Department of Mathematics, Stanford University, Stanford, CA 94305-2125, USA \\ ${ }^{2}$ Department of Mathematics, University of California, Santa Cruz, CA 95064, USA
}

\section{Introduction}

It is known (see [A] and [EM]) that the group of volume preserving diffeomorphisms of a compact oriented Riemannian manifold is the configuration space of incompressible ideal hydrodynamics. The group of symplectic diffeomorphisms plays a similar role in plasma physics (see [MW]). Both groups carry a structure of metric space, natural from the point of view of mechanics, which can be defined, starting with the $L^{2}$-norm on their Lie algebras, as the infimum of length of paths connecting two points (see $\$ 2$ for exact definitions). Shnirelman in [Sh] has proved that the group of volume-preversing diffeomorphisms of the cube in $\mathbb{R}^{3}$ has finite diameter and has announced the result that this is false for the square.

Despite the fact that Shnirelman formulated his theorem only for the 3dimensional cube, his proof can be modified for the case of the group of volume preserving diffeomorphisms of any compact simply-connected Riemannian manifold of dimension $>2$. However, the diameter can become infinite if the fundamental group is not trivial (see Appendix).

It turns out that the situation with the group of symplectic diffeomorphisms is completely different. We prove in this paper that the diameter of the symplectomorphism group of any compact exact symplectic manifold (necessarily with boundary) is infinite. We think that the result is true without the assumption of the exactness of the symplectic form.

It is important to point out that, as a contrary to the volume-preserving case, infiniteness of the diameter of the group of symplectomorphisms has local nature and has nothing to do with the topology of the underlying manifold. The distinction between the cases comes from the fact that the fundamental group of the group of linear symplectic transformations is infinite while in the volume preserving case it is finite in dimension $>2$. 
Although the structure of metric space on the group of symplectomorphisms $\mathscr{D}_{\omega}$ considered in this paper is natural from the point of view of continuum mechanics, there exist other metrics which are quite natural from the geometric point of view. In fact, we prove in this paper infiniteness of the diameter in the metric on $\mathscr{D}_{\omega}$ generated by the $L^{1}$ - (rather than the $L^{2}$-) norm on its Lie algebra. This implies, of course, the result for all $L^{p}$-norms, $p \geqq 1$. Moreover, the group $\mathscr{D}_{\omega}$ admits even more interesting metrics. One of them which is actually biinvariant and can be canonically defined in terms of the symplectic structure was recently discovered by $\mathrm{H}$. Hofer (see [H]). Infiniteness of the diameter of $\mathscr{D}_{\omega}$ in Hofer's metric can also be seen within the philosophy of our paper. While the main ingredient in our proof is the Calabi invariant (which is a volume-like symplectic invariant), the proof for Hofer's metric requires more subtle symplectic invariants - Ekeland-Hofer-Zehnder's capacities or Gromov's width.

The paper is organized as follows. In Sect. 2 we define the metric on $\mathscr{D}_{\omega}$ and state the main theorem. Section 3 contains a reduction to the case of exact symplectomorphisms. Section 4 is devoted to a general inequality which we use in the Sect. 5 to prove the main results. In the Appendix we give a brief explanation of the role of the fundamental group in the volume preserving case.

Note that the proof can be greatly simplified for the case of the group of exact symplectomorphisms fixed at the boundary of the manifold. In this case the result follows (see [ER]) from three main ingredients: an inequality between the length of a path and the value of the Calabi invariant of the resulted symplectomorphism; the independence of the Calabi invariant from the choice of the path; the existence of symplectomorphisms with arbitrarily large values of the Calabi invariant.

The proof in the general case requires more work. But even in the special case above the approach of this paper has some advantages. It shows that not only $\mathscr{D}_{\omega}$ but all its non-trivial normal subgroups have infinite diameter (see Theorem 2.2 below).

\section{Statement of the results}

\subsection{Metrics on the group of volume preserving diffeomorphisms}

Let $M$ be a compact manifold (possibly with boundary) with a volume form $\mu$. Fix a Riemannian metric on $M$. Let $\mathscr{D}$ be the connected component of the identity in the group of volume preserving diffeomorphisms of $(M, \mu)$. A tangent vector to $\mathscr{D}$ at a point $\eta$ is a map $X_{\eta}: M \rightarrow T M$ such that $X_{\eta}(x) \in T_{\eta(x)}(M), x \in M$ (see [EM]). Let us put $\left\|X_{\eta}\right\|_{p}=\left(\iint_{M}\left\|X_{\eta}(x)\right\|^{p} \mu\right)^{\frac{1}{p}}$, where the norm $\left\|X_{\eta}(x)\right\|$ is taken with respect to the chosen Riemannian on $M$. Note that $\left\|X_{\eta}\right\|_{p} \geqq c_{p}\left\|X_{\eta}\right\|_{1}$ for a positive constant $c_{p}$.

Given a path $\eta_{t} \in \mathscr{D}, t \in[0,1]$, we define its $p$-length $l_{p}\left(\left\{\eta_{t}\right\}\right)$ by the formula

$$
l_{p}\left(\left\{\eta_{t}\right\}\right)=\int_{0}^{1}\left\|\frac{d \eta_{t}}{d t}\right\|_{p} d t
$$

The length function $l_{p}$ defines a distance function $d_{p}$ on $\mathscr{D}$ by $d_{p}(f, g)=\inf l_{p}\left(\left\{\eta_{t}\right\}\right)$, where the infimum is taken over all paths $\eta_{t}, t \in[0,1]$, joining $\eta_{0}=f$ and $\eta_{1}=g$. 
For any pathwise connected subset $A \subset \mathscr{D}$ we define its $p$-diameter by the formula

$$
\operatorname{diam}_{p}(A)=\sup _{f, g \in A} d_{p}(f, g) .
$$

For a pair $A \subset B$ in $\mathscr{D}$ we define a relative $p$-distance by the formula

$$
d_{p}(f, g \mid B)=\inf l_{p}\left(\left\{\eta_{t}\right\}\right)
$$

where the infimum is taken over all paths $\eta_{t} \in B, t \in[0,1]$, joining $\eta_{0}=f, \eta_{1}=g \in A$. The relative $p$-diameter of $A$ in $B$ is now defined by the formula

Note that

$$
\operatorname{diam}(A \mid B)=\sup _{f, g \in A} d_{p}(f, g \mid B) .
$$

$$
\operatorname{diam}_{p}(A \mid B) \geqq c_{p} \operatorname{diam}_{1}(A \mid B) .
$$

In this paper we are proving infiniteness of diameters of certain subgroups of $\mathscr{D}$. According to the inequality above the strongest results are with respect to diam . $_{1}$. From now on we will consider only this case and we will drop the index "I" from our notations.

\subsection{Groups of symplectomorphisms}

Let $M$ be a compact exact symplectic manifold (necessarily with boundary) with the symplectic form $\omega=d \lambda$. Then $\mu=\omega^{n}$ is a volume form on $M$. Let us denote by $\mathscr{D}_{\omega}, \mathscr{D}_{\omega} \subset \mathscr{D}$, the identity component of the group of symplectomorphisms of $M$, i.e. diffeomorphisms $f: M \rightarrow M$ with $f^{*} \omega=\omega$.

A symplectomorphism $f \in \mathscr{D}_{\omega}$ is called exact if the closed 1 -form $\lambda_{f}=f^{*} \lambda-\lambda$ is exact. The exactness of $\lambda_{f}$ is independent on the choice of $\lambda$ with $d \lambda=\omega$. Let $\mathscr{D}_{\omega}^{e}$ be the subgroup of $\mathscr{D}_{\omega}$ which consists of exact symplectomorphisms. Note that $\mathscr{D}_{\omega}^{e}$ is path connected (see 3.5.2 below). By $\mathscr{Q}_{\omega, c}^{e}$ we will denote the subgroup of $\mathscr{D}_{\omega}^{e}$ which consists of diffeomorphisms which are fixed near the boundary $\partial M$.

Calabi in $[\mathrm{Ca}]$ defined an epimorphism

$$
\mathrm{Cal}: \mathscr{D}_{\omega, \partial}^{\boldsymbol{e}} \rightarrow \mathbb{R}
$$

see 5.1 below.

The kernel of this homomorphism which we will denote by $\mathscr{D} \omega, 0,0$ consists of symplectomorphisms with zero Calabi invariant. According to Banyaga (see [B]) $\mathscr{D}_{\omega, \partial}^{e, 0}$ is the commutator $\left[\mathscr{Q}_{\omega, \theta}^{e}, \mathscr{D}_{\omega, \theta}^{e}\right]$ and it is a simple group.

Theorem 2.2.1.

$$
\operatorname{diam}\left(\mathscr{D}_{\omega}\right)=+\infty
$$

Theorem 1 is a direct corollary of the following more precise result:

Theorem 2.2.2.

$$
\operatorname{diam}\left(\mathscr{D}_{\omega, \mathrm{a}}^{e, 0} \mid \mathscr{D}_{\omega}\right)=+\infty
$$




\section{Reduction to the exact case}

\subsection{Exact symplectomorphisms}

Let $(M, \omega=d \lambda)$ be an exact compact symplectic manifold. A vector field $X$ on $M$ is called symplectic if its dual 1 -form $\theta=X] \omega$ is closed which is equivalent to $\mathscr{L}_{X} \omega=0$. If $\theta$ is exact then $X$ is called Hamiltonian. If $\eta_{t}, t \in[0,1]$, is a path in $\mathscr{D}_{\omega}$ then for any $t \in[0,1]$ the vector field $X_{t}(x)=\frac{d \eta_{t}\left(\eta_{t}^{-1}(x)\right.}{d t}$ is symplectic. All paths in $\mathscr{D}_{\omega}$ which we will consider in this paper, will start at the identity and we will usually assume that without mentioning it. We will call the path $\eta_{t}, t \in[0,1]$, Hamiltonian if the field $X_{t}$ is Hamiltonian for all $t \in[0,1]$. The function $H_{t}, t \in[0,1]$, with $d H_{t}=\theta_{t}$ is called, in this case, the (time-dependent) Hamiltonian which generates $\eta_{t}, t \in[0,1]$. The function $H_{t}$ is determined up to adding a constant $C_{t}$.

The following standard fact relates the notions of exact symplectomorphisms and Hamiltonian paths.

Lemma 3.1.1. The path $\eta_{t}, t \in[0,1]$ is Hamiltonian if and only if it consists of exact symplectomorphisms.

Indeed,

$$
\left.\eta_{t}^{*} \lambda-\lambda=\int_{0}^{t} \frac{d \eta_{\tau}^{*}(x)}{d \tau} d \tau=\int_{0}^{t} \eta_{t}^{*}\left(X_{\tau}\right] w\right) d \tau+d \int_{0}^{t} \eta_{t}^{*}\left(\lambda\left(x_{\tau}\right)\right) d \tau
$$

Thus $\eta_{t}^{*} \lambda-\lambda$ is exact for all $t \in[0,1]$ if and only if the form $\left.\theta_{t}=X_{t}\right\rfloor w$ is exact for all $t \in[0,1]$.

\subsection{The characteristic line bundle}

The restriction $\left.\omega\right|_{\partial M}$ has 1-dimensional kernel $K \subset T(\partial M)$ which is called the characteristic line bundle of $\partial M$. A symplectic field $X$ on $M$ is tangent to $\partial M$ if and only if the form $\theta=X] \omega$ vanhishes on $K$. In this case $X$ integrates to a flow $X^{t}: M \rightarrow M, t \in \mathbb{R}$, such that $X^{t}(\partial M)=\partial M, t \in \mathbb{R}$. Let us denote by $H_{K}^{1}(M ; \mathbb{R})$ the subspace of $H^{1}(M ; \mathbb{R})$ which consists of cohomology classes of closed 1 -forms vanishing on $K$.

\subsection{Special flows}

For a closed 1-form $\alpha$ we denote by [ $\alpha$ ] its cohomology class.

Let us fix closed 1 -forms $\theta_{1}, \ldots, \theta_{N}$ vanishing on $K$ and such that $\left[\theta_{1}\right], \ldots,\left[\theta_{N}\right]$ form a basis of $H_{K}^{1}(M ; \mathbb{R})$. Let $\partial_{k}$ be the symplectic field dual to the forms $\theta_{k}$, $k=1, \ldots, N$, namely, $\left.\theta_{k}=\partial_{k}\right] \omega$.

Take continuous functions $A_{1}, \ldots, A_{N}:[0,1] \rightarrow \mathbb{R}$ with $A_{k}(0)=0, k=1, \ldots, N$ and define a vector field $A(t), t \in[0,1]$, by the formula

$$
A(t)=\sum_{k=1}^{N} A_{k}(t) \partial_{k}
$$

Let $A^{u}(t), u \in \mathbb{R}$, be the flow generated by $A(t)$, for a fixed $t \in[0,1]$. Thus

$$
\frac{d A^{u}(t)}{d u}=A(t) \circ A^{u}(t)
$$




\section{Lemma 3.3.1.}

$$
\left[\left(A^{u}(t)\right) * \lambda-\lambda\right]=u \sum_{k=1}^{N} A_{k}(t)\left[\theta_{k}\right]
$$

Proof. We have (comp. 3.1.1):

$$
\begin{aligned}
{\left[\left(A^{u}(t)\right)^{*} \lambda-\lambda\right] } & \left.=\int_{0}^{u}\left[\left(A^{u}(\tau)\right)^{*}(A(\tau)] \omega\right)\right] d \tau \\
& \left.\left.=\int_{0}^{u}[A(\tau)] \omega\right] d \tau=u[A(\tau)] \omega\right] \\
& =u \sum_{k=1}^{N} A_{k}(t)\left[\theta_{k}\right] . \quad \square
\end{aligned}
$$

The following lemma 3.3.2 immediately follows from compactness arguments.

Lemma 3.3.2. Let $D=\max \left(\left|A_{k}(t)\right|\right), k=1, \ldots, N, t \in[0,1]$. Then there exists a $C=C(D)$ such that $\left\|d A^{u}(t)(x)\right\| \leqq C(D)$ for any $t \in[0,1], x \in M,|u| \leqq 1$.

Lemma 3.3.3. Suppose that $\int_{0}^{1}\left|\frac{d A_{k}(t)}{d t}\right| d t \leqq D^{\prime}$. Then there exists a constant $C^{\prime}=C^{\prime}\left(D^{\prime}\right)$ such that $l\left(\left\{A^{u}(t)\right\}\right) \leqq C^{\prime}\left(D^{\prime}\right)$ for $|u| \leqq 1$.

Proof. Let us embed the compact manifold with boundary $M$ isometrically in an Euclidean space. From

we conclude that

$$
\begin{aligned}
A^{u}(t)(x)-x & =\int_{0}^{u} \frac{d}{d s}\left(A^{s}(t)(x)\right) d s \\
& =\int_{0}^{u} A(t)\left(A^{s}(t)(x)\right) d s
\end{aligned}
$$

$$
\begin{aligned}
\frac{d A^{u}(t)(x)}{d t}= & \int_{0}^{u} A^{\prime}(t)\left(A^{s}(t)(x)\right) d s \\
& +\int_{0}^{u} d A(t)\left(A^{s}(t)(x)\right)\left(\frac{d A^{s}(t)(x)}{d t}\right) d s
\end{aligned}
$$

and hence

$$
\begin{aligned}
& \int_{0}^{1}\left\|\frac{d A^{u}(t)(x)}{d t}\right\| d t \leqq \int_{0}^{1} \int_{0}^{u} \| A^{\prime}(t)\left(A^{s}(t)(x) \| d s d t\right. \\
& +\int_{0}^{1} \int_{0}^{u} \| d A(t)\left(A^{s}(t)(x)\|\| \frac{d A^{s}(t)(x)}{d t} \| d s d t .\right.
\end{aligned}
$$

However, since $A(t)=\sum_{k=1}^{N} A_{k}(t) \partial_{k}$, it follows that $\left\|A^{\prime}(t)(y)\right\| \leqq C_{1} \max _{k=1, \ldots, N}\left|A_{k}^{\prime}(t)\right|$, for any $y \in M$, where the constant $C_{1}$ depends only on $M$ and the vector fields $\partial_{k}$, $k=1, \ldots, N$. Thus the first term in the previous inequality is bounded above by

$$
C_{1} \max _{k=1, \ldots, N} \int_{0}^{1}\left|A_{k}^{\prime}(t)\right| d t \leqq C_{1} D^{\prime}
$$


By compactness of $M,\|d A(t)(y)\| \leqq C_{2} \max _{k=1, \ldots, N}\left|A_{k}(t)\right|$, for all $y \in M$, where the constant $C_{2}$ depends only on the vector fields $\partial_{k}, k=1, \ldots, N$. But

$$
\left|A_{k}(t)\right| \leqq \int_{0}^{1}\left|A_{k}^{\prime}(s)\right| d s \leqq D^{\prime}, \quad \text { i.e. }
$$

for all $t \in[0,1]$ and all $k=1, \ldots, N$. Therefore,

Thus we have

$$
\|d A(t)(y)\| \leqq C_{2} D^{\prime} .
$$

$$
\int_{0}^{1}\left\|\frac{d A^{u}(t)(x)}{d t}\right\| d t \leqq C_{1} D^{\prime}+C_{2} D^{\prime} \int_{0}^{u}\left[\int_{0}^{1}\left\|\frac{d A^{s}(t)(x)}{d t}\right\| d t\right] d s .
$$

By Gronwall's inequality we conclude

$$
\int_{0}^{1}\left\|\frac{d A^{u}(t)(x)}{d t}\right\| d t \leqq C_{1} D^{\prime} e^{C_{2} D^{\prime}}=\tilde{C}\left(D^{\prime}\right)
$$

Therefore

$$
l\left(\left\{A^{u}(t)\right\}\right)=\int_{0}^{1} \int_{M}\left\|\frac{d A^{u}(t)(x)}{d t}\right\| \mu(x) d t \leqq C^{\prime}\left(D^{\prime}\right)
$$

Remark 3.3.4. The functions $C(D)$ and $C^{\prime}(D)$ in 3.3 .2 and 3.3.3 can obviously be chosen monotonically increasing with $D$.

\subsection{Estimates on the length of a non-Hamiltonian path}

Let $\eta_{t}, t \in[0,1]$, be a path in $\mathscr{D}_{\omega}$ (starting at $\eta_{0}=i d$, see 3.1). Let $X_{t}(x)=\frac{d \eta_{t}}{d t}\left(\eta_{t}^{-1}(x)\right.$ ) and $\alpha_{t}$ be the closed 1 -form $\omega$-dual to $X_{t}, t \in[0,1]$.

Lemma 3.4.1. The cohomology classes of the 1-forms $\eta_{t}^{*} \lambda-\lambda$ and $\int_{0}^{t} \alpha_{s} d s$ coincide. In particular, $\left[\eta_{t}^{*} \lambda-\lambda\right] \in H_{K}^{1}(M)$.

Proof.

$$
\left[\eta_{t}^{*} \lambda-\lambda\right]=\int_{0}^{t} \eta_{s}^{*}\left[\alpha_{s}+d\left(\lambda\left(X_{s}\right)\right)\right] d s=\int_{0}^{t}\left[\alpha_{s}\right] d s .
$$

However, $\alpha_{t}$ annihilates the characteristic line bundle $K$ since $X_{t}$ is necessarily tangent to $\partial M$. Therefore

$$
\int_{0}^{t}\left[\alpha_{s}\right] d s \in H_{K}^{1}(M) .
$$

Let $\eta_{t}, X_{t}$ and $\alpha_{t}$ be as above. Let $\left[\theta_{1}\right], \ldots\left[\theta_{N}\right]$ be the basis of $H_{K}^{1}(M)$ considered in 3.3. Then $\left[\eta_{t}^{*} \lambda-\lambda\right]=\sum_{1}^{N} A_{k}(t)\left[\theta_{k}\right]$.

\section{Lemma 3.4.2.}

$$
l\left(\left\{\eta_{t}\right\}\right) \geqq Q \max _{k} \int_{0}^{1}\left|A_{k}^{\prime}(t)\right| d t
$$


The following lemma 3.4.3 is a key ingredient in the proof of 3.4.2.

Lemma 3.4.3. Let $S \subset M$ be an embedded circle and $\Gamma, S \subset \Gamma \subset M$, be its tubular neighborhood. Then for any closed 1-form $\theta$ on $M$ we have

$$
C\left|\int_{C} \theta\right| \leqq \int_{\Gamma}\|\theta\| \mu
$$

for a constant $C$ independent on $\theta$.

Proof. Let $\chi: S \times \mathscr{D}^{2 n-1} \rightarrow \Gamma$ be a decomposition of the tubular neighborhood $\Gamma$ and $\frac{\partial}{\partial t}$ be a unit tangent vector field along $S^{1}$. Then

$$
\begin{aligned}
& \int_{\Gamma}\|\theta\| \mu \geqq C_{1} \int_{\Gamma} \mid \theta\left(d_{x}\left(\frac{\partial}{\partial t}\right)\left|\mu \geqq C_{1}^{\prime} \int_{\mathscr{D}^{2 n-1} \times S^{1}}\right| \chi^{*}(\theta)\left(\frac{\partial}{\partial t}\right) \mid d^{2 n-t} x d t\right. \\
& \geqq C_{1}^{\prime} \int_{\mathscr{D}^{2 n-1}}\left|\int_{x \times S^{1}} \chi^{*}(\theta)\left(\frac{\partial}{\partial t}\right) d t\right| d^{2 n-1} x \\
&=C_{1}^{\prime} \int_{\mathscr{D}^{2 n-1}}\left|\int_{S} \theta\right| d^{2 n-1} x=C\left|\int_{S} \theta\right| \cdot \quad \square
\end{aligned}
$$

Proof of 3.4.2. Let us choose disjoint embedded circles $S_{1}, \ldots, S_{l} \subset M$ such that their homology classes $\left[S_{1}\right], \ldots\left[S_{l}\right]$ form a basis of $H_{1}(M)$. Choose disjoint tubular neighborhoods $\Gamma_{i} \supset S_{i}, i=1, \ldots, l$. Let us complete the basis $\left[\theta_{1}\right], \ldots\left[\theta_{N}\right]$ of $H_{K}^{1}(M) \subset H^{1}(M)$ to a basis $\left[\theta_{1}\right], \ldots,\left[\theta_{N}\right], \ldots,\left[\theta_{l}\right]$ of $H^{1}(M)$. Let $B=\left(b_{i j}\right)$ be a $(l \times l)$-matrix $b_{i j}=\int_{S_{j}} \theta_{i}$. Then $B$ is nondegenerate and, therefore, $\|B y\| \geqq b\|y\|$ for $y \in \mathbb{R}^{l}, b>0$. We have

$$
\begin{aligned}
l\left(\left\{\eta_{t}\right\}\right) & =\int_{0}^{1} \int_{M}\left\|X_{t}\left(\eta_{t}(x)\right)\right\| \mu d t \\
& \geqq \int_{0}^{1} \sum_{k=1}^{1} \int_{\Gamma_{k}}\left\|\alpha_{t}(x)\right\| \mu d t .
\end{aligned}
$$

Applying 3.4.3 and 3.4.1 we get

$$
\begin{aligned}
l\left(\left\{\eta_{t}\right\}\right) \geqq C_{1} \int_{0}^{1} \sum_{k=1}^{l}\left|\int_{S_{k}} \alpha_{t}\right| d t \\
=C_{1} \int_{0}^{1} \sum_{k=1}^{l}\left|\sum_{j=1}^{N} A_{j}^{\prime}(t) \int_{S_{k}} \theta_{j}\right| d t \\
=C_{1} \int_{0}^{1} \sum_{k=1}^{l}\left|\sum_{j=1}^{N} b_{j k} A_{j}^{\prime}(t)\right| d t \geqq C_{2} \int_{0}^{1}\|B y\| d t
\end{aligned}
$$

where $y=\left(A_{1}^{\prime}(t), \ldots, A_{N}^{\prime}(t), 0, \ldots, 0\right) \in \mathbb{R}^{l}$. Therefore

$$
l\left(\left\{\eta_{t}\right\}\right) \geqq C_{2} b \int_{0}^{1}\|y\| d t \geqq Q \max _{k} \int_{0}^{1}\left|A_{k}^{\prime}(t)\right| d t
$$




\subsection{Reduction to the exact case}

In this section we continue to use the notations which were introduced above. In particular, $\eta_{t}, t \in[0,1]$, is a path in $\mathscr{D}_{\omega}, X_{t}$ is the corresponding symplectic vector field, $\alpha_{t}$ is $\omega$-dual to $X_{t}, t \in[0,1]$. We denote by $\left[\theta_{1}\right] \ldots\left[\theta_{N}\right]$ a basis in $H_{K}^{1}(M)$ and by $\partial_{1}, \ldots, \partial_{N}$ symplectic fields dual to $\theta_{1}, \ldots, \theta_{N}$. We define functions $A_{1}, \ldots, A_{N}:[0,1] \rightarrow \mathbb{R}$ by the formula $\left[\eta_{t}^{*} \lambda-\lambda\right]=\sum_{k=1}^{N} A_{k}(t)\left[\theta_{k}\right]$. We keep the notation $A(t)$ for the vector field $\sum_{k=1}^{N} A_{k}(t) \partial_{k}, t \in[0,1]$, and denote by $A^{u}(t), u \in \mathbb{R}$, the flow generated by $A(t)$.

Lemma 3.5.1. Let $D=\max _{k} \int_{0}^{1}\left|A_{k}^{\prime}(t)\right| d t$. Suppose that $\eta_{1}$ is an exact symplectomorphism. Let $\psi_{t}=A^{-1}(t) \circ \eta_{t}, t \in[0,1]$. Then the path $\left\{\psi_{t}\right\}$ is Hamiltonian, $\psi_{1}=\eta_{1}$ and

$$
l\left(\left\{\psi_{t}\right\}\right) \leqq C(D) l\left(\left\{\eta_{t}\right\}\right)+C^{\prime}(D)
$$

for increasing functions $C(D)$ and $C^{\prime}(D)$.

Proof. First note that $\left[\psi_{t}^{*} \lambda-\lambda\right]=\left[\left(A^{-1}(t)\right)^{*} \lambda-\lambda\right]+\left[\eta_{t}^{*} \lambda-\lambda\right]=0$ according to 3.3.1. Therefore, $\left\{\psi_{t}\right\}$ is Hamiltonian by 3.1.1. Now we have

$$
\begin{aligned}
l\left(\left\{\psi_{t}\right\}\right) & =\int_{0}^{1} \int_{M}\left\|\frac{d \psi_{t}(x)}{d t}\right\| \mu d t \\
& \leqq \int_{0}^{1} \int_{M}\left(\left\|\frac{d A^{-1}(t)}{d t}\left(\eta_{t}(x)\right)\right\|+\left\|d A^{-1}(t)\left(X_{t}\left(\eta_{t}(x)\right)\right)\right\|\right) \mu d t \\
& =l\left(\left\{A^{-1}(t)\right\}\right)+\int_{0}^{1} \int_{M}\left\|d A^{-1}(t)\left(X_{t}(x)\right)\right\| \mu d t .
\end{aligned}
$$

Applying 3.3.2 and 3.3.3, we get

$$
l\left(\left\{\psi_{t}\right\}\right) \leqq C^{\prime}(D)+C(D) l\left(\left\{\eta_{t}\right\}\right) .
$$

Finally note that $A(1)=0$ because $\eta_{1}$ is exact. Therefore $\psi_{1}=\eta_{1}$.

The following lemma 3.5.2 is the main result of this section.

Lemma 3.5.2. Let $\mathscr{D}_{\omega}^{e}$ be the subgroup of $\mathscr{D}_{\omega}$ which consists of exact xmplectomorphisms $M \rightarrow M$. Then there exists an increasing function $\varphi: \mathbb{R}_{+} \rightarrow \mathbb{R}$ such that $d\left(i d, f \mid \mathscr{D}_{\omega}^{e}\right) \leqq \varphi\left(d\left(i d, f \mid \mathscr{D}_{\omega}\right)\right)$ for any $f \in \mathscr{D}_{\omega}$.

Proof. Take a diffeomorphism $f=\eta_{1} \in \mathscr{D}_{\omega}^{e}$. Let $L=d\left(i d, f \mid \mathscr{D}_{\omega}\right)$. Then $\eta_{1}$ can be joined with the identity by a path $\eta_{t} \in \mathscr{D}_{\omega}$ with $l\left(\left\{\eta_{t}\right\}\right)<2 L$. Applying the construction from 3.5.1 we can find a Hamiltonian path $\psi_{t}, t \in[0,1]$, with $\psi_{1}=\eta_{1}$ such that

$$
l\left(\left\{\psi_{t}\right\}\right) \leqq C(D) l\left\{\eta_{t}\right\}+C^{\prime}(D) \leqq 2 C(D) L+C^{\prime}(D) .
$$

But Lemma 3.4.2 implies that

$$
D \leqq \frac{1}{Q} l\left\{\eta_{t}\right\} \leqq \frac{2 L}{Q} .
$$


Therefore,

or

$$
l\left(\left\{\psi_{t}\right\}\right) \leqq 2 C\left(\frac{2 L}{Q}\right) L+C^{\prime}\left(\frac{2 L}{Q}\right)=\varphi(L)
$$

$$
d\left(i d, f \mid \mathscr{D}_{\omega}^{e}\right) \leq \varphi\left(d\left(i d, f \mid \mathscr{D}_{\omega}^{e}\right) .\right.
$$

Remark 3.5.3. Lemma 3.5.1 implies, in particular, that the group $\mathscr{D}_{\omega}^{e}$ is pathconnected (see Section 2).

\section{An inequality}

The goal of this section is the following inequality 4.1 .

Proposition 4.1. Let $\mu$ be a compact Riemannian manifold (with or without boundary) with volume form $\mu, H: M \rightarrow \mathbb{R}$ be a smooth function. Then

$$
\int_{M} \int_{M}|H(x)-H(y)| \mu_{x} \mu_{y} \leqq C \int_{M}\|\nabla H\| \mu .
$$

Proof. Let us start with the case when $M$ is a ball $B$ of radius 1 in $\mathbb{R}^{n}$. Fix any nonzero vector $z \in \mathbb{R}^{n}$. Let us denote by $\Pi_{z}: \mathbb{R}^{n} \rightarrow \mathbb{R}^{n}$ a parallel translation $\Pi_{z}(x)=x-z$. Let $B_{z}=B \cap \Pi_{z}(B)$ and $D_{z}$ be the image of $B_{z}$ under the orthogonal projection $\pi: B \rightarrow L$ onto the hyperplane $L \subset \mathbb{R}^{n}$ which is perpendicular to $z$. For a point $u \in D_{z}$ we denote by $l_{u}$ a fiber $\pi^{-1}(u) \subset B$ over the point $u \in D_{z}$. Let $l_{u}^{\prime}=l_{u} \cap B_{z}$. So a point $y \in B_{z} \subset B$ has coordinates $u \in D_{z}, t \in l_{u}$. Then we have

$$
\begin{aligned}
\int_{B_{z}}|H(y+z)-H(y)| d^{n} y & =\int_{D_{z}} \int_{i_{u}}|H(y+z)-H(y)| d t d^{n-1} u \\
& \leqq \int_{D_{z}} \int_{l_{u}} \int_{i_{u}}\left|\frac{d H}{d \tau}(\tau, u)\right| d \tau d t d^{n-1} u \\
& \leqq \int_{D_{z}} \int_{i_{u}} \int_{i_{u}}\|\nabla H(\tau, u)\| d \tau d t d^{n-1} u \\
& \leqq 2 \int_{D_{z}} \int_{i_{u}}\|\nabla H(\tau, u)\| d \tau d^{n-1} u \\
& =2 \int_{B}\|\nabla H(y)\| d^{n} y
\end{aligned}
$$

Therefore,

$$
\begin{aligned}
& \int_{B} \int_{B}|H(x)-H(y)| d^{n} x d^{n} y \\
& \int_{B} \int_{B_{z}}|H(y+z)-H(y)| d^{n} y d^{n} z \\
& \quad \leqq \int_{B} \int_{B_{z}}\|\nabla H(y)\| d^{n} y d^{n} z \leqq 2 \operatorname{vol}(B) \int_{B}\|\nabla H(y)\| d^{n} y
\end{aligned}
$$


Now consider the general case. Take embeddings $f_{1}, \ldots, f_{N}: B \rightarrow M$ such that $M \times M=\bigcup_{1}^{N} f_{i}(B) \times f_{i}(B)$. Then

$$
\begin{aligned}
\int_{M} \int_{M}|H(x)-H(y)| \mu_{x} \mu_{y} & \leqq \sum_{1}^{N} \int_{f_{i}(B)} \int_{f_{i}(B)}|H(x)-H(y)| \mu_{x} \mu_{y} \\
& \leqq C_{1} \sum_{1}^{N} C \int_{B} \int_{B}\left|H\left(f_{i}(u)\right)-H\left(f_{i}(v)\right)\right| d^{n} u d^{n} v \\
& \leqq C_{2} \sum_{1}^{N} \int_{B}\left\|\nabla\left(H \circ f_{i}\right)(u)\right\| d^{n} u \\
& \leqq C_{3} \sum_{1}^{N} \int_{f_{i}(B)}\|\nabla H\| \mu \leqq C_{4} \sum_{1}^{N} \int_{M}\|\nabla H\| \mu \\
& =C \int_{M}\|\nabla H\| \mu .
\end{aligned}
$$

\section{Exact case}

Let now $(M, \omega=d \lambda)$ be a compact exact symplectic manifold.

\subsection{Calabi invariant}

Let $\eta \in \mathscr{D}_{\omega, \partial}^{e}$ be an exact symplectomorphism which is fixed near $\partial M$. Then the form $\lambda_{\eta}=\eta^{*} \lambda-\lambda$ is exact and is 0 near $\partial M$. Let $h: M \rightarrow \mathbb{R}$ be a function with $\left.h\right|_{\partial M}=0$, $d h=\lambda_{\eta}$. Then the number $\int_{M} h \mu$ does not depend on the choice of the primitive $\lambda$ of $\omega$ (see $[\mathrm{C}]$ and $[\mathrm{B}]$ ) and is called the Calabi invariant of $\eta$;

$$
\operatorname{Cal}(\eta)=\int_{M} h \mu .
$$

The function Cal defines an epimorphism of the group $\mathscr{D}_{\omega, \partial}^{e}$ onto $\mathbb{R}$.

Let Fix $(\eta) \subset M$ be the set of fixed points of $\eta$ and let $U \subset$ Fix $(\eta)$ be the component of Fix $(\eta)$ which contains the boundary $\partial M$. Fix a point $x \in U$ and for any $y \in M$ let us choose a path $\gamma_{x y}$ which starts at $x$ and ends at $y$. Then

and, therefore,

$$
h(y)=\int_{\eta\left(\gamma_{x y}\right)} \lambda-\int_{\gamma_{x y}} \lambda
$$

$$
\operatorname{Cal}(\eta)=\int_{M}\left(\int_{\eta\left(\gamma_{x y}\right)} \lambda-\int_{\gamma_{x y}} \lambda\right) \mu
$$

\subsection{An identity}

Let $\eta_{t}, t \in[0,1]$ be a Hamiltonian path in $\mathscr{D}_{\omega}$ with $\eta_{0}=i d, \eta_{1}=\eta$ and let $H_{t}: M \rightarrow \mathbb{R}, t \in[0,1]$ be its generating Hamiltonian, $\left.d H_{t}=X_{t}\right] \omega$.

Lemma 5.2.1. Let $\gamma$ be a path in $M$ joining two points $x, y \in M$. Then

$$
\int_{\eta(\gamma)} \lambda-\int_{\gamma} \lambda=\int_{0}^{1} \lambda\left(X_{t}\left(\eta_{t}(y)\right) d t-\int_{0}^{1} \lambda\left(X_{t}\left(\eta_{t}(x)\right) d t+\int_{0}^{1}\left(H_{t}\left(\eta_{t}(y)\right)-H_{t}\left(\eta_{t}(x)\right)\right) d t .\right.\right.
$$


Proof. Let $K=[0,1] \times[0,1] \subset \mathbb{R}^{2}$. Consider a map $S: K \rightarrow M$ defined by the formula $S(u, t)=\eta_{t}(\gamma(u))$, then

but

$$
\int_{\mathbf{K}} S^{*} w=\int_{\partial \mathbf{K}} S^{*} \lambda=\int_{\gamma} \lambda+\int_{\left\{\eta_{t}(y)\right\}} \lambda-\int_{\eta(\gamma)} \lambda-\int_{\left\{\eta_{t}(x)\right\}} \lambda,
$$

$$
\begin{aligned}
\int_{K} S^{*} w & =\int_{K} w\left(d \eta_{t}\left(\frac{d \gamma(u)}{d u}\right), X_{t}\left(\eta_{t}(\gamma(u))\right)\right) d u d t \\
& =-\int_{K} d\left(H_{t} \circ \eta_{t}\right)(\gamma(u))\left(\frac{d \gamma(u)}{d u}\right) d u d t \\
& =-\int_{0}^{1}\left(H_{t}\left(\eta_{t}(y)\right)\right)-H_{t}\left(\eta_{t}(x)\right) d t .
\end{aligned}
$$

Therefore,

$$
\int_{\eta(y)} \lambda-\int_{y} \lambda=\int_{0}^{1} \lambda\left(X_{t}\left(\eta_{t}(y)\right) d t-\int_{0}^{1} \lambda\left(X_{z}\left(\eta_{t}(X)\right) d t+\int_{0}^{1}\left(H_{t}\left(\eta_{t}(y)\right)-H_{t}\left(\eta_{t}(x)\right)\right) d t\right.\right.
$$

\subsection{Exact symplectomorphisms and Calabi invariant}

Let us fix an embedded ball $B \subset \operatorname{Int} M$ and a smaller ball $B_{0} \subset$ Int $B$. Let $A=B \backslash$ Int $B_{0}$. We will call a symplectomorphism $f: M \rightarrow M$ special if $f(B)=B$ and $\left.f\right|_{A}=i d$. For a diffeomorphism $h: B \rightarrow B$ which is fixed near $\partial B$ we denote as in 2.2 and 5.1 by $\mathrm{Cal}(h)$ its Calabi invariant.

Proposition 5.3.1. Let $\eta_{t}, t \in[0,1]$, be a Hamiltonian path in $\mathscr{D}_{\omega}$ such that $\eta=\eta_{1}$ is special. Then $l\left(\left\{\eta_{t}\right\}\right)>K \mathrm{Cal}\left(\left.\eta\right|_{\mathrm{B}}\right)$ where the constant $K$ is independent on the choice of path $\left\{\eta_{i}\right\}$.

Proof. Let us fix a point $x \in A$ and for any $x \in B$ let us choose a path $\gamma_{x y}$ joining the points $x, y \in B$ we have

Applying now 5.2 we get

$$
\operatorname{Cal}\left(\left.\eta\right|_{B}\right)=\int\left(\int_{B} \lambda-\int_{\gamma_{x y}} \lambda\right) \mu_{y}
$$

$$
\begin{aligned}
\operatorname{Cal}\left(\left.\eta\right|_{B}\right)= & \int_{B}\left[\int_{0}^{1} \lambda\left(X_{t}\left(\eta_{t}(y)\right)\right) d t-\int_{0}^{1} \lambda\left(X_{t}\left(\eta_{t}(x)\right)\right) d t\right. \\
& +\int_{0}^{1}\left(H_{t}\left(\eta_{t}(y)\right)-H_{t}\left(\eta_{t}(x)\right) d t\right] \mu_{y} .
\end{aligned}
$$

Integrating both parts of the inequality over $A$ we get

$$
\begin{aligned}
\operatorname{Vol}(A) \operatorname{Cal}\left(\left.\eta\right|_{B}\right)= & \operatorname{Vol}(A) \int_{B} \int_{0}^{1} \lambda\left(X_{t}\left(\eta_{t}(y)\right) d t \mu_{x}\right. \\
& -\operatorname{Vol}(B) \iint_{A}^{1} \lambda\left(X_{t}\left(\eta_{t}(x)\right) d t \mu_{x}\right. \\
& +\int_{0}^{1} \int_{A} \int_{B}\left(H _ { t } \left(\eta_{t}(y)-H_{t}\left(\eta_{t}(x)\right) d t \mu_{y} \mu_{x} .\right.\right.
\end{aligned}
$$


Thus

$$
\begin{aligned}
\operatorname{Vol}(A)\left|\operatorname{Cal}\left(\left.\eta\right|_{B}\right)\right| \leqq & C\left[\operatorname{Vol}(A) \int_{0}^{1} \int_{M}\left\|X_{t}\right\| d t \mu+\operatorname{Vol}(B) \int_{0}^{1} \int_{M}\left\|X_{t}\right\| d t \mu\right] \\
& +\int_{0}^{1} \int_{M} \int_{M} \mid H_{t}\left(\eta_{t}(x)\right)-H_{t}\left(\eta_{t}(y) \mid \mu_{x} \mu_{y}\right. \\
= & C(\operatorname{Vol}(A)+\operatorname{Vol}(B)) l\left(\left\{\eta_{t}\right\}\right) \\
& +\int_{0}^{1} \int_{M} \int_{M}\left|H_{t}(x)-H_{t}(y)\right| \mu_{x} \mu_{y} .
\end{aligned}
$$

Now we apply the inequality 4.1 and get

$$
\left|\operatorname{Cal}\left(\left.\eta\right|_{B}\right)\right| \leqq C_{1} l\left(\left\{\eta_{t}\right\}\right)+C_{2} \int_{0}^{1} \int_{M}\left\|\nabla H_{t}\right\| \mu d t=\left(C_{1}+C_{2}\right) l\left(\left\{\eta_{t}\right\}\right) .
$$

\section{Proof of Theorem 2.2.2}

We start with the following simple lemma.

Lemma 6.1. Let $B \subset M$ be an embedded ball as in 5.2. Let $h: B \rightarrow B$ be a symplectomorphism fixed near the boundary $\partial B$ and isotopic to the identity. Then $h$ can be extended to a symplectomorphism $g: M \rightarrow M$ fixed near $\partial M$, isotopic to the identity and such that.

$$
\operatorname{Cal}(g)=0 .
$$

Proof. Let us choose a ball $B_{1} \subset \operatorname{Int}(M \backslash B)$. Take a symplectomorphism $h_{1}: B_{1} \rightarrow B_{1}$ fixed near $\partial B_{1}$ which is isotopic to the identity and such that Cal $\left(h_{1}\right)=-\mathrm{Cal}(h)$. The required symplectomorphism $g: M \rightarrow M$ is equal to $h$ on $B, h_{1}$ on $B_{1}$ and is the identity on $M \backslash\left(B_{1} \cup B\right)$.

Recall that $\mathscr{D}_{\omega}^{e}$ denotes the group of exact symplectomorphisms.

\section{Proposition 6.2.}

$$
\operatorname{diam}\left(\mathscr{D}_{\omega, 0}^{e, 0} \mid \mathscr{D}_{\omega}^{e}\right)=+\infty .
$$

Proof. The Calabi homomorphism is surjective. Therefore, for any $N>0$ there exists a symplectomorphism $h_{N}: B \rightarrow B$ with $\mathrm{Cal}\left(h_{N}\right)>N$ which is isotopic to the identity. (The required $h_{N}$ can be, for example, constructed as the time-one map of the Hamiltonian flow generated by a Hamiltonian $H: B \rightarrow \mathbb{R}$ with $H=0$ near $\partial B$ and $\int_{B} H \mu>N$.) Extend $h_{N}$ using 6.1 to a symplectomorphism $g_{N} \in \mathscr{D}_{\omega, \hat{\partial}}$. Take a Hamiltonian path $\eta_{t} \in \mathscr{D}_{\omega}^{e}, t \in[0,1]$ with $\eta_{0}=i d, \eta_{1}=g_{N}$. According to 5.3.1 $l\left(\left\{\eta_{t}\right\}\right)$ $>K N$. Hence $d\left(i d, g_{N}\right)=\inf _{\left\{\eta_{t}\right\}} l\left(\left\{\eta_{t}\right\}\right) \geqq K N$. Therefore, $\quad \operatorname{diam}\left(\mathscr{D}_{\omega, \partial}^{e, 0} \mid \mathscr{D}_{\omega}^{e}\right)=$ $+\infty$. 


\section{Appendix}

As we mentioned in the Introduction, the diameter of the group of the volume preserving diffeomorphisms of a manifold is finite when the manifold is simply connected (see [Sh]). We will explain in this section that in the non-simplyconnected case the diameter can be infinite.

Theorem A.1. Let $M$ be a manifold with a volume form $\mu$ and $\mathscr{D}$ be (the identity component of) the group of volume preserving diffeomorphisms of $M$. Suppose that $H^{1}(M ; \mathbb{R}) \neq 0$ and $\pi_{1}(M)$ has trivial center. Then

$$
\operatorname{diam}(\mathscr{D})=\infty \text {. }
$$

Proof. Let a 1 -form a represent a nontrivial cohomology class from $H^{1}(M ; \mathbb{R})$. Let $g$ be the Lie algebra of divergent free vector fields. The formula $\mathscr{A}(X)=\int_{M} a(X) \mu$, $X \in g$, defines $\mathscr{A}$ as a 1 -form on $\mathscr{D}$. A straightforward computation shows that this is a closed 1-form (comp. [Fa]) i.e., for any contractible loop $\eta$ in $\mathscr{D}$ we have $\int \mathscr{A}=0$. In fact this form is exact if the evaluation homomorphism $\pi_{1}(\mathscr{D}) \rightarrow H_{1}(\stackrel{\pi}{M} ; \mathbb{R})$ is trivial (see [McD]). For example, this is so if the center of $\pi_{1}(M)$ is trivial. Then the formula

$$
\mathscr{P}(x)=\int_{\eta_{x}} \mathscr{A},
$$

where $x \in \mathscr{D}$ and $\eta_{x}$ is a path connecting $i d$ with $x$ defines a functional on $\mathscr{D}$ which is a primitive of $\mathscr{A}$. This is the so-called mass flow homomorphism (see [Fa]). It is easy to see that $\mathscr{F}$ is not trivial and, therefore, takes arbitrarily large values.

Now note that

$$
\begin{aligned}
|\mathscr{F}(x)| & =\left|\int_{\eta_{x}} \mathscr{A}\right|=\left|\int_{0}^{1}\left(\int_{M} a\left(\frac{d \eta_{x}(t)}{d t}\right) \mu\right) d t\right| \\
& \leqq C \int_{0}^{1} \int_{M}\left\|\frac{d \eta_{x}(t)}{d t}\right\| \mu d t \leqq \mathcal{C} l\left(\eta_{x}\right)
\end{aligned}
$$

where $l\left(\eta_{x}\right)$ is the length of the path $\eta_{x}$ for the metric we consider in this paper $($ see $(2-2))$.

Hence,

$$
d(i d, x) \geqq \widetilde{C}|\mathscr{F}(x)|
$$

Therefore, $d(i d, x)$ takes arbitrarily large values together with $\mathscr{F}(x)$ which means that $\operatorname{diam}(\mathscr{D})=\infty$.

Acknowledgements. We learned about Shnirelman's theorem from V.I. Arnold during his visit to MSRI in May 1989. He also conjectured that in the symplectic case the situation is different. We would like to thank him for useful discussions. Many people contributed useful ideas towards our understanding of the problem. We are grateful to all of them and especially to N. A'Campo and C. McMullen. We thank A. Fathi and D. McDuff from whom we learned about the mass flow homomorphism. We want to thank our referee who indicated a mistake in the original version of the paper. This paper was essentially completed when both authors were members of the Mathematical Sciences Research Institute at Berkeley. We would like to thank MSRI for its hospitality. 


\section{References}

[A] Arnold, V.I. : Sur la géométrie différentielle des groupes de Lie de dimension infinie et ses applications à l'hydrodynamique des fluids parfaits. Ann. Inst. Fourier, Grenoble 16, 319-361 (1966)

[B] Banyaga, A. : Sur la structure de groupe des difféomorphismes qui préservent une forme symplectique. Comment. Math. Helv. 53, 174-227 (1978)

[C] Calabi, E.: On the group of automorphisms of a symplectic manifold. In: Gunning, R. (ed.) Problems in Analysis, Symposium in honor of S. Bochner, Princeton University Press, 1970, pp. 1-26

[ER] Eliashberg, Ya., Ratiu, T.: Note on the diameter of the group of symplectomorphisms of the ball, Travaux de Séminaire Sud-Rhodanien de Geometrie, 1989 (to appear)

[EM] Ebin, D., Marsden, J.: Groups of diffeomorphisms and the motion of an incompressible fluid. Ann. Math. 92, 102-163 (1970)

[Fa] Fathi, A.: Structure of the group of homeomorphisms preserving a good measure on a compact manifold. Ann. Sci. Ec. Norm. Super 13, 45-93 (1980)

[H] Hofer, H.: On the topological properties of symplectic maps. 1989 (preprint)

[MW] Marsden, J., Weinstein, A.: The Hamiltonian structure of the Maxwell-Vlasov equations, Physica, 4D, 394-406 (1982)

[McD] McDuff, D.: Symplectic diffeomorphisms and flux homomorphism. Invent. Math. 77, 353-365 (1984)

[Sh] Shnirelman, A.L.: On the geometry of the group of diffeomorphisms and the dynamics of an ideal incompressible fluid, Mat. Sbornik $128(170), 1,1985$, English translation, Math. USSR Sbornik 56, 79-105 (1987) 